\title{
BMJ Open Palliative care for people who use substances during communicable disease epidemics and pandemics: a scoping review protocol
}

\author{
Daniel Z Buchman (D , , ${ }^{1,2,3}$ Philip Ding, ${ }^{4}$ Samantha Lo, ${ }^{4}$ Naheed Dosani, ${ }^{5,6}$ \\ Rouhi Fazelzad, ${ }^{7}$ Andrea D Furlan, ${ }^{8,9,10}$ Sarina R Isenberg, ${ }^{11,12}$ Sheryl Spithoff, ${ }^{13,14}$ \\ Alissa Tedesco, ${ }^{6,15}$ Camilla Zimmermann (D) , 4,9,16,17 Jenny Lau ${ }^{4,14,16}$
}

To cite: Buchman DZ, Ding P, Lo S, et al. Palliative care for people who use substances during communicable disease epidemics and pandemics: a scoping review protocol. BMJ Open 2021;11:e053124. doi:10.1136/ bmjopen-2021-053124

- Prepublication history and additional supplemental material for this paper are available online. To view these files, please visit the journal online (http://dx.doi.org/10.1136/ bmjopen-2021-053124).

Received 05 May 2021 Accepted 12 0ctober 2021

Check for updates

(c) Author(s) (or their employer(s)) 2021. Re-use permitted under CC BY-NC. No commercial re-use. See rights and permissions. Published by BMJ.

For numbered affiliations see end of article.

Correspondence to Dr Daniel Z Buchman; daniel.buchman@utoronto.ca

\section{ABSTRACT}

Introduction Communicable disease epidemics and pandemics magnify the health inequities experienced by marginalised populations. People who use substances suffer from high rates of morbidity and mortality and should be a priority to receive palliative care, yet they encounter many barriers to palliative care access. Given the pre-existing inequities to palliative care access for people with life-limiting illnesses who use substances, it is important to understand the impact of communicable disease epidemics and pandemics such as COVID-19 on this population.

Methods and analysis We will conduct a scoping review and report according to the Preferred Reporting Items for Systematic Reviews and Meta-Analyses extension for Scoping Reviews reporting guidelines. We conducted a comprehensive literature search in seven bibliographical databases from the inception of each database to August 2020. We also performed a grey literature search to identify the publications not indexed in the bibliographical databases. All the searches will be rerun in April 2021 to retrieve recently published information because the COVID-19 pandemic is ongoing at the time of this writing. We will extract the quantitative data using a standardised data extraction form and summarise it using descriptive statistics. Additionally, we will conduct thematic qualitative analyses and present our findings as narrative summaries.

Ethics and dissemination Ethics approval is not required for a scoping review. We will disseminate our findings to healthcare providers and policymakers through professional networks, digital communications through social media platforms, conference presentations and publication in a scientific journal.

\section{INTRODUCTION}

Communicable disease epidemics and pandemics magnify the healthcare inequities encountered by people who use substances. ${ }^{1}$ The United Nations 2019 World Drug Report estimated that 271 million people used substances in the previous year. Among this population, $13 \%$ suffered from substance use disorders (SUDs), ${ }^{2}$ which are most commonly

\section{Strengths and limitations of this study}

- This study will be the first scoping review of peerreviewed and grey literature on providing palliative care to people who use substances during communicable disease epidemics and pandemics.

- Our scoping review will follow the established Levac et afs six-stage framework, which builds on the contributions of Arksey and O'Malley, Joanna Briggs Institute guidance document for the conduct of scoping reviews, and the Preferred Reporting Items for Systematic Reviews and Meta-Analyses extension for Scoping Reviews reporting guidelines.

- This review provides a means to include the high volume of rapid publications during the first year of the COVID-19 pandemic.

- Our search may be limited because the review cannot include all possible literature, as new information regarding treatment changes daily and will not include literature published in languages other than English and French.

related to alcohol, cannabis and opioids. ${ }^{3}$ In 2017, there were 585000 deaths and 42 million years of healthy life lost globally as a result of substance use. ${ }^{2}$ People who use substances are at high risk of life-limiting illnesses such as HIV, cancers, strokes and liver cirrhosis. ${ }^{3}$ The most common causes of non-overdose-related deaths among people with opioid use disorders are cancers $(17 \%-$ $29 \%)$, cerebrovascular diseases $(17 \%-22 \%)$, pulmonary diseases $(20 \%)$ and infectious diseases $(14 \%){ }^{45}$ The novel coronavirus pandemic (COVID-19) has increased the risks associated with substance use, and vice versa. For instance, the pandemic has created immense challenges of providing care for people who use substances, ${ }^{6}$ and loss of traditional in-person clinical and support interventions may exacerbate substance use and lead to worse health outcomes, particularly 
those who are structurally disadvantaged. ${ }^{7}$ An analysis of electronic health records in the USA found that people recently diagnosed with a SUD were at an increased risk of COVID-19 and had a higher prevalence of comorbid chronic diseases affecting the kidneys, liver, lung, as well as cardiovascular diseases, type 2 diabetes, obesity and cancer. ${ }^{8}$ The overdose crisis has worsened during COVID19 , with some scholars referring to 'an epidemic within a pandemic'. ${ }^{9}$ Given the high morbidity and mortality associated with substance use, the frequent and concurrent negative impacts of social and structural determinants of health, and the resulting increased risk during communicable disease emergencies including the current COVID-19 pandemic, these individuals should be a priority population to receive palliative care.

Palliative care addresses the suffering experienced by people with life-limiting illnesses through symptom management, psychosocial support and advance care planning. ${ }^{10}$ Demand for palliative care is likely to increase during a pandemic due to increased mortality, with the additional possibility of deprioritising individuals with a lower chance of survival for critical care if available resources are overwhelmed. ${ }^{11}$ Many people who use substances encounter barriers to receiving palliative care, including structural inequities resulting in fewer social supports and a lack of financial resources. ${ }^{12}$ Moreover, delivery of community-based health services, including palliative care, may be restricted due to concerns that the settings are risky or unsafe. ${ }^{12}$ Zero-tolerance policies toward non-medical use of substances may also restrict access to palliative care units and hospices.

For example, in response to the high transmissibility and mortality of SARS-CoV-2, healthcare has been limited to essential services. ${ }^{13}$ These services include the provision of substance use treatment and outreach, but many programmes, such as supervised consumption sites, are closed or have reduced hours of operation. ${ }^{14}$ Similarly, palliative care providers are considered essential but their care has transitioned to primarily virtual care for clinically stable patients to reduce physical meetings. ${ }^{15}$ However, people may not have access to the necessary technologies to receive virtual care (eg, smartphones, internet), especially those who have severe mental health disorders, poor health literacy and socioeconomic disadvantages. ${ }^{14}$ These changes are necessary to combat the COVID-19 pandemic but may have disproportionate negative consequences for people who use substances. ${ }^{16}$ In combination with the overdose crisis, restricted access to mental health and substance use services can increase the risk of relapse and overdoses. ${ }^{14} 17$ Additionally, physical distancing is an important public health measure to mitigate the spread of COVID-19 but this measure may not be possible for some people who use substances, and especially for individuals who live in congregate settings such as shelters. Physical isolation can reduce their support system and further increase their risk of relapse and overdose. ${ }^{17}$ Given the pre-existing inequities to palliative care access, it is important to understand the impact of communicable disease epidemics and pandemics on people with lifelimiting illnesses who use substances.

\section{Rationale}

Preliminary database searches (PubMed, Web of Science) in May 2020 did not identify any reviews that address the palliative care needs for people who use substances during communicable disease outbreaks, such as severe acute respiratory syndrome (SARS), influenza A (H1N1), Ebola and COVID-19. Therefore, we propose a knowledge synthesis project that will allow us to identify and map available and emerging evidence about palliative care for patients who use substances during communicable disease epidemics and pandemics. The findings from our scoping review will provide accessible and relevant evidence for healthcare professionals and decisionmakers (eg, policymakers, administrators) that can be applied to the COVID-19 pandemic response efforts.

\section{Review objective}

The primary objective of this scoping review is to answer the research question, 'what is known about communicable disease epidemics and pandemics, palliative care and people who use substances?' Specifically, the focus of our review is on people with life-limiting illnesses who use substances. Scoping review research questions are intended to be broad, as comprehensiveness and breadth are important to map a literature. ${ }^{18}$

\section{METHODOLOGY}

This article describes the protocol for a scoping review that is currently being conducted. Our scoping review is guided by Levac et al s six-stage framework, ${ }^{19}$ which builds on the contributions of Arksey and O'Malley, ${ }^{20}$ Joanna Briggs Institute guidance document for the conduct of scoping reviews, ${ }^{21}$ and the Preferred Reporting Items for Systematic Reviews and Meta-Analyses extension for Scoping Reviews (PRISMA-ScR) ${ }^{22}$ reporting guidelines.

\section{Eligibility criteria}

We will use the PECOS (Population, Exposure, Comparison or Control, Outcomes and Characteristics) framework $^{23}$ to structure our evidence eligibility criteria. For 'Population', we will include adults (defined as $\geq 18$ years of age) who use substances and have life-limiting illnesses (eg, advanced cancer, end-stage organ failure), including those who receive treatment for SUDs and use prescription drugs and/or unregulated substances. In our study, we will acknowledge that substance use occurs on a spectrum $^{24}$ and that for some people, their substance use may not be a 'problem' in their lives although they might meet the diagnostic criteria for SUD as per the Diagnostic and Statistical Manual of Mental Disorders, fifth edition. We will focus on the most used substances globally: alcohol, tobacco, cannabis, opioids (eg, heroin), cocaine, central nervous system depressants (eg, benzodiazepines) and stimulants (eg, amphetamines). ${ }^{25}$ The 'Exposure' will be 
communicable disease outbreaks, specifically epidemics and pandemics (eg, COVID-19, SARS). We will include literature with and without 'Comparison or Control' groups, including grey literature (eg, government reports, policy statements). Our 'Outcomes' of interest will be quantitative outcomes and qualitative themes related to palliative care; specifically, interventions, access to services, policies/guidelines and clinical programmes that address physical, psychological, social and/or spiritual issues faced by patients with life-limiting illnesses and their families. We will include topics such as education outreach to family members and harm reduction and overdose prevention if they occur within a palliative care framework. Lastly, our study 'Characteristics' will include research conducted in any country and published in English or French. We will restrict our studies to these languages due to limited available resources and the rapid nature of our review. We aim to include peer-reviewed and non-peer-reviewed literature including letters to the editors, theses and book chapters. Eligible studies will not be limited to specific publication years.

\section{Information sources and search strategy}

An information specialist, in collaboration with our team of technical and subject matter experts in scoping reviews, palliative care and substance use, developed a comprehensive search strategy to identify studies in the following bibliographical databases: Medline ALL (Medline and Epub Ahead of Print and In-Process and Other NonIndexed Citations), Embase Classic+Embase, Cochrane Database of Systematic Reviews, Cochrane Central Register of Controlled Trial, PsycInfo all from the OvidSP platform and Scopus from Elsevier. Where available, both controlled vocabulary terms and text words were used. There were no date or publication type restrictions. Where applicable, the search was limited to adults and the English/French language. The online supplemental appendix presents our Ovid Medline (R) ALL 1946 to 28 August 2020, search as an example of our search strategy. We are using EndNote, a reference management software, to store data from these database searches. ${ }^{26}$

We also conducted a grey literature search in August 2020 to identify published literature that is not indexed in the bibliographical databases, including but not limited to guidelines, conference and meeting abstracts, theses and reports. We searched grey literature resources, such as TRIP, Google Scholar, Google, WHO, websites of prominent health organisations and associations of palliative care, public health (communicable disease), substance use, individual conference, theses and guidelines. Search strategies were customised based on each resource's available searching feature. For instance, Google limits the number of queries; therefore, individual searches were conducted for each communicable disease listed in the study's scope combining with palliative care and substance use. Each search was further limited to a specific country to retrieve more focused results.
All the searches from the bibliographical databases and grey literature resources will be rerun in April 2021 to retrieve recently published literature. Furthermore, we will hand search reference lists of all potentially eligible full-text publications to identify additional relevant studies.

\section{Methods limitations}

Our search may be limited because the scoping review cannot include all possible literature, as new information regarding treatment changes daily and will not include literature published in languages other than English and French. Since our review explores different types of communicable diseases (eg, COVID-19, HIV, tuberculosis), specific disease may have different palliative care resource implications at the health system level and may look different depending on the type of substance in question. It is beyond the scope of this protocol to explore all possible permutations and combinations.

\section{Selection of sources of evidence}

From our database search from inception to 28 August 2020, we imported 5939 records obtained from the database searches into Covidence, a web-based literature review software. ${ }^{27}$ We will use this program to detect and remove duplicates. We will screen all the record titles and abstracts in duplicate according to our eligibility criteria. The same process will be applied to the full-text articles. If there is a disagreement, we will consult a third party to act as a tiebreaker. Records with no abstract will be screened based only on their title. If there are conference proceedings or abstracts without full text, we will exclude these records due to the limited information available. These three main reasons will count toward exclusion: wrong population (eg, people with curative diagnoses), wrong exposure (eg, non-communicable disease public health emergencies) and wrong outcomes (eg, not related to palliative care).

We have imported the 2889 records identified in the initial grey literature search into Google Sheets. We will manually assess each record's eligibility by applying our PECOS framework criteria. If there are disagreements between the two investigators assessing the records, they will be resolved by consulting a third member as a tiebreaker.

\section{Data charting process}

For our research from August 2020, we developed a standardised data charting form using Google Sheets to extract the following variables: author/organisation, publication year, journal, country of the corresponding author, article type (commentary/opinion article, conference/workshop, guideline, news article, programme report, other), publication language, countries and settings in which studies were conducted (acute care, inpatient palliative care, long-term care, community, home care, infectious diseases clinic, palliative care clinic), populations (lifelimiting diagnoses, substances used, sex and gender, age, 


\begin{tabular}{|c|c|c|c|}
\hline Code & Definition/description & Examples & References \\
\hline Intervention & $\begin{array}{l}\text { '...an approach that improves the quality of life of patients and their } \\
\text { families facing the problem associated with life-threatening illness, } \\
\text { through the prevention and relief of suffering by means of early } \\
\text { identification and impeccable assessment and treatment of pain and } \\
\text { other problems, physical, psychosocial and spiritual.' }\end{array}$ & $\begin{array}{l}\text { Pain } \\
\text { management, } \\
\text { symptom } \\
\text { management, } \\
\text { caregiver } \\
\text { support }\end{array}$ & $\begin{array}{l}\text { https://www.who.int/cancer/ } \\
\text { palliative/definition/en/ } \\
\text { https://www.canada.ca/en/ } \\
\text { health-canada/services/palliative- } \\
\text { care.html }\end{array}$ \\
\hline Policies/guideline & $\begin{array}{l}\text { '...decisions, plans and actions that are undertaken to achieve } \\
\text { specific health care goals within a society.' } \\
\text { 'An explicit health policy can achieve several things: it defines a } \\
\text { vision for the future which in turn helps to establish targets and points } \\
\text { of reference for the short and medium term. It outlines priorities and } \\
\text { the expected roles of different groups; and it builds consensus and } \\
\text { informs people.' }\end{array}$ & $\begin{array}{l}\text { Policies that } \\
\text { limit opioid } \\
\text { prescribing, } \\
\text { policies that } \\
\text { prevent people } \\
\text { who use drugs } \\
\text { from accessing } \\
\text { hospice care. }\end{array}$ & $\begin{array}{l}\text { https://www.who.int/topics/ } \\
\text { health_policy/en/ }\end{array}$ \\
\hline Access & $\begin{array}{l}\text { '...systems, processes and resources [that] are in place so that } \\
\text { people with identified palliative care needs can receive palliative care } \\
\text { support whenever they need it' } \\
\text { 'Access to appropriate palliative care means that it meets the needs } \\
\text { of those requiring care at the right time, in the right place and by the } \\
\text { right provider.' } \\
\text { WHO's definition of access for universal health coverage: } \\
\text { '...availability of good health services... and other aspects of service } \\
\text { organization and delivery that allow people to obtain the services } \\
\text { when they need them'; '... measure of people's ability to pay for } \\
\text { services without financial hardship'; and '... people's willingness to } \\
\text { seek services' }\end{array}$ & $\begin{array}{l}\text { Virtual } \\
\text { appointments, } \\
\text { interpreters }\end{array}$ & $\begin{array}{l}\text { https://www.hqontario.ca/ } \\
\text { portals/0/documents/evidence/ } \\
\text { quality-standards/qs-palliative- } \\
\text { care-clinical-guide-en.pdf } \\
\text { https://www.cihi.ca/sites/default/ } \\
\text { files/document/access-palliative- } \\
\text { care-2018-en-web.pdf } \\
\text { https://www.who.int/bulletin/ } \\
\text { volumes/91/8/13-125450.pdf }\end{array}$ \\
\hline
\end{tabular}

ethnicities), target audiences (healthcare professionals, researchers, policymakers, governments), communicable diseases and palliative care outcomes (interventions, access to services, policies/guidelines and clinical programmes).

\section{Synthesis of results}

We will develop a PRISMA-ScR flow diagram to map out the review process including the number of identified records, studies included and excluded, and the reasons for exclusion. We will also provide a narrative description of the search decision process. Quantitative data (eg, study characteristics documented in our data charting process) will be analysed using descriptive statistics and summarised in tabular and graphical forms. We will summarise the findings in text using a narrative approach. Knowledge gaps will be identified through comparative analysis of the extracted data, particularly the palliative care outcomes.

We will analyse the qualitative data thematically to help us make sense of the large amounts of data. Based on the priorities of our knowledge users, who are experts in palliative care and addictions, we have developed a codebook with the following four palliative care-related codes that will be applied to our findings: interventions, clinical programmes, policies/guidelines and access (please see table 1). We will code each record in duplicate using the codebook and the qualitative analysis software, NVivo. ${ }^{28}$ During the coding process, we will use the constant comparative method to inductively identify additional codes that capture common themes and differences within and between the records. ${ }^{29}$ Based on our inductive thematic analysis, we will likely identify subthemes within each main code. Unlike the deductive component of our analysis, the subthemes cannot be identified a priori. We will report our findings in narrative form, and our synthesis will explore relationships such as the type of substance use and life-limiting illness, as well as geographical, cultural factors, as well as epidemic or pandemic context in which the palliative care is provided.

\section{Patient and public involvement}

There were no patients involved in the design of this protocol. The research team includes healthcare professionals who provide palliative care for people who use substances and several team members who work closely with people who use substances in the context of hospital and community-based work. Our research team also includes a principal knowledge user, Canadian Virtual Hospice, that provides comprehensive online knowledge about palliative and end-oflife care. These members have provided guidance on 
designing the scoping review and will help lead our knowledge dissemination strategy.

\section{ETHICS AND DISSEMINATION}

We did not require approval from a research ethics board for this study as it does not involve human participants or unpublished secondary data. We will refine our research question and interpret our results in consultation with our content experts and knowledge users. Our principal knowledge user, Canadian Virtual Hospice, is an award-winning digital health palliative care organisation that provides comprehensive online knowledge about palliative and end-of-life care. Their website has more than 25000 visits per month. ${ }^{30}$ They will lead our integrated and end-of-grant knowledge mobilisation strategy. Our target audiences will be healthcare professionals, decision-makers, patients and their families. The findings from this scoping review will be disseminated through professional networks, digital communications using social media platforms, conference presentations and scientific journal publications. Our knowledge dissemination plan includes development of infographics, digital communications (eg, Twitter) and online news features.

\section{Implications}

Our scoping review will provide important information to inform palliative care practice and policy. We aim to rapidly synthesise available evidence to identify strengths and gaps in the existing knowledge about palliative care for people who use substances during communicable disease epidemics and pandemics. Our goal is to provide information that may be used to minimise inequities in palliative care access for this population, especially during times of health system strain and resource scarcity.

\section{Author affiliations}

${ }^{1}$ Bioethics, Centre for Addiction and Mental Health, Toronto, Ontario, Canada ${ }^{2}$ Clinical Public Health, Dalla Lana School of Public Health, University of Toronto, Toronto, Ontario, Canada

${ }^{3}$ Joint Centre for Bioethics, University of Toronto, Toronto, Ontario, Canada

${ }^{4}$ Department of Supportive Care, University Health Network, Toronto, Ontario, Canada

${ }^{5}$ Department of Medicine, Unity Health Toronto, Toronto, Ontario, Canada

${ }^{6}$ Inner City Health Associates, Toronto, Ontario, Canada

${ }^{7}$ Library and Information Services, Princess Margaret Cancer Centre, University Health Network, Toronto, Ontario, Canada

${ }^{8}$ Toronto Rehab, University Health Network, Toronto, Ontario, Canada

${ }^{9}$ Department of Medicine, Temerty Faculty of Medicine, University of Toronto, Toronto, Ontario, Canada

${ }^{10}$ Institute for Work \& Health, University Health Network, Toronto, Ontario, Canada

${ }^{11}$ Bruyere Research Institute, Ottawa, Ontario, Canada

${ }^{12}$ Department of Medicine, University of Ottawa, Ottawa, Ontario, Canada

${ }^{13}$ Women's College Hospital, Toronto, Ontario, Canada

${ }^{14}$ Department of Family and Community Medicine, Temerty Faculty of Medicine,

University of Toronto, Toronto, Ontario, Canada

${ }^{15}$ Sinai Health System, Toronto, Ontario, Canada

${ }^{16}$ Division of Palliative Care, University Health Network, Toronto, Ontario, Canada

${ }^{17}$ Division of Palliative Medicine, Temerty Faculty of Medicine, University of Toronto, Toronto, Ontario, Canada
Twitter Daniel Z Buchman @DanielZBuchman and Andrea D Furlan @adfurlan

Collaborators Shelly Cory.

Contributors The scoping review protocol was drafted by $\mathrm{JL}$ and $\mathrm{DB}$, and then edited and reviewed by PD, ND, RF, AF, SRI, SL, SS, AT and CZ.

Funding This work was supported by the Canadian Institute of Health Research (grant number CMS-171746).

Disclaimer The funding organisation had no role in the preparation, review approval or data analyses of the course content.

Competing interests AF receives an unrestricted educational grant to maintain the online opioid self-assessment programme from the Canadian Generic Pharmaceutical Association (CGPA). AF is the inventor of the App Opioid Manager, which is sold in iTunes. The app is owned by UHN, the hospital where AF works and AF does not receive any profits from the app sales. AF has a YouTube monetised channel and receives payment for advertisements on the channel. There are some videos related to opioids in her channel. All other authors report no competing interests.

Patient consent for publication Not required.

Provenance and peer review Not commissioned; externally peer reviewed.

Supplemental material This content has been supplied by the author(s). It has not been vetted by BMJ Publishing Group Limited (BMJ) and may not have been peer-reviewed. Any opinions or recommendations discussed are solely those of the author(s) and are not endorsed by BMJ. BMJ disclaims all liability and responsibility arising from any reliance placed on the content. Where the content includes any translated material, BMJ does not warrant the accuracy and reliability of the translations (including but not limited to local regulations, clinical guidelines, terminology, drug names and drug dosages), and is not responsible for any error and/or omissions arising from translation and adaptation or otherwise.

Open access This is an open access article distributed in accordance with the Creative Commons Attribution Non Commercial (CC BY-NC 4.0) license, which permits others to distribute, remix, adapt, build upon this work non-commercially, and license their derivative works on different terms, provided the original work is properly cited, appropriate credit is given, any changes made indicated, and the use is non-commercial. See: http://creativecommons.org/licenses/by-nc/4.0/.

ORCID iDs

Daniel Z Buchman http://orcid.org/0000-0001-8944-6647

Camilla Zimmermann http://orcid.org/0000-0003-4889-0244

\section{REFERENCES}

1 Dainius P. Statement by the un expert on the right to health* on the protection of people who use drugs during the COVID-19 pandemic. United Nations, 2020. Available: https://www.ohchr.org/EN/ NewsEvents/Pages/DisplayNews.aspx?NewsID=25797\&LangID=E

2 World drugs report 2019, 2019. Available: https://wdr.unodc.org/ wdr2019/prelaunch/WDR19_Booklet_2_DRUG_DEMAND.pdf

3 Degenhardt L, Charlson F, Ferrari A, et al. The global burden of disease attributable to alcohol and drug use in 195 countries and territories, 1990-2016: a systematic analysis for the global burden of disease study 2016. Lancet Psychiatry 2018;5:987-1012.

4 Hser YI, Mooney LJ, Saxon AJ. High mortality among patients with opioid use disorder in a large healthcare system. J Addict Med 2017.

5 Bech AB, Clausen T, Waal $\mathrm{H}$, et al. Mortality and causes of death among patients with opioid use disorder receiving opioid agonist treatment: a national register study. BMC Health Serv Res 2019;19:440.

6 Ornell F, Moura HF, Scherer JN, et al. The COVID-19 pandemic and its impact on substance use: implications for prevention and treatment. Psychiatry Res 2020;289:113096.

7 Melamed OC, Hauck TS, Buckley L, et al. COVID-19 and persons with substance use disorders: inequities and mitigation strategies. Subst Abus 2020;41:286-91.

8 Wang QQ, Kaelber DC, Xu R. COVID-19 risk and outcomes in patients with substance use disorders: analyses from electronic health records in the United States. Mol Psychiatry 2020.

9 Alexander GC, Stoller KB, Haffajee RL, et al. An epidemic in the midst of a pandemic: opioid use disorder and COVID-19. Ann Intern Med 2020;173:57-8.

10 World Health Organization. Palliative care, 2021. Available: https:// www.who.int/health-topics/palliative-care [Accessed 7 Apr 2021]. 
11 Arya A, Buchman S, Gagnon B, et al. Pandemic palliative care: beyond ventilators and saving lives. Can Med Assoc $J$ 2020;192:E400-4.

12 McNeil R, Guirguis-Younger M. Illicit drug use as a challenge to the delivery of end-of-life care services to homeless persons: perceptions of health and social services professionals. Palliat Med 2012;26:350-9.

13 Government of Canada. Guidance on essential services and functions in Canada during the COVID-19 pandemic, 2021. Available: https://www.publicsafety.gc.ca/cnt/ntnl-scrt/crtcl-nfrstrctr/esf-sfe-en. aspx [Accessed 7 Apr 2021].

14 Alexander GC, Stoller KB, Haffajee RL, et al. An epidemic in the midst of a pandemic: opioid use disorder and COVID-19. Ann Intern Med 2020;173:57-8.

15 Hannon B, Mak E, Al Awamer A, et al. Palliative care provision at a tertiary cancer center during a global pandemic. Support Care Cancer 2021;29:2501-7.

16 Volkow ND. Collision of the COVID-19 and addiction epidemics. Ann Intern Med 2020;173:61-2.

17 Dong K, Meador K, Hyshka E. Supporting people who use substances in acute care settings during the COVID-19 pandemic: CRISM - Interim Guidance Document, 2020.

18 Colquhoun $\mathrm{HL}$, Levac D, O'Brien KK, et al. Scoping reviews: time for clarity in definition, methods, and reporting. $J$ Clin Epidemiol 2014;67:1291-4.

19 Levac D, Colquhoun H, O'Brien KK. Scoping studies: advancing the methodology. Implement Sci 2010;5:69.

20 Arksey H, O'Malley L. Scoping studies: towards a methodological framework. Int J Soc Res Methodol 2005;8:19-32.
21 Peters MDJ, Godfrey CM, Mclnerney P. Methodology for JBI scoping reviews. The Joanna Briggs Institute Reviewers' Manual 2015, 2015.

22 Tricco AC, Lillie E, Zarin W, et al. PRISMA extension for scoping reviews (PRISMA-ScR): checklist and explanation. Ann Intern Med 2018;169:467-73.

23 Morgan RL, Whaley P, Thayer KA, et al. Identifying the PECO: a framework for formulating good questions to explore the association of environmental and other exposures with health outcomes. Environ Int 2018;121:1027-31.

24 Mahmoud KF, Finnell D, Savage CL, et al. A concept analysis of substance misuse to inform contemporary terminology. Arch Psychiatr Nurs 2017;31:532-40.

25 National Institute on Drug Abuse. Commonly used drugs charts, 2020. https://www.drugabuse.gov/drug-topics/commonly-useddrugs-charts

26 EndNote. Clarivate analytics. Available: https://endnote.com/ [Accessed 7 Nov 2018].

27 Covidence. Better systematic review management. Available: https:// www.covidence.org/home [Accessed 7 Nov 2018]

28 NVIVO. NVIVO, 2020. Available: https://www.qsrinternational.com/ nvivo-qualitative-data-analysis-software/about/nvivo [Accessed 23 Nov 2020].

29 Fram S. The constant comparative analysis method outside of grounded theory. Qual Rep 2013;18:1-25.

30 Canadian Virtual Hospice. Canadian virtual hospice. Available: http:// www.virtualhospice.ca/en_US/Main+Site+Navigation/Home.aspx [Accessed 27 Apr 2021]. 